\title{
Comparação do poder de correção do instrumental de Luque- Galveston e do parafuso pedicular no tratamento cirúrgico da escoliose neuromuscular
}

\author{
Comparison between deformity correction of Luque-Galveston
} instrumentation and pedicle screw fixation in the surgical treatment of neuromuscular scoliosis

\section{Comparación del poder de corrección del instrumental de Luque- Galveston y del tornillo pedicular en el tratamiento quirúrgico de la escoliosis neuromuscular}

\author{
Murilo Tavares Daher ${ }^{1}$ \\ Paulo Tadeu Maia Cavali² \\ Marcus Alexandre Mello Santo² \\ Alexander Junqueira Rossato ${ }^{2}$ \\ Mauricio Antonelli Lehoczki \\ Élcio Landim ${ }^{3}$
}

\section{RESUMO}

Objetivo: avaliar o poder de correção do parafuso pedicular em comparação ao sistema de Luque-Galveston no tratamento cirúrgico da escoliose neuromuscular. Métodos: foram avaliados 74 pacientes submetidos à artrodese exclusivamente pela via posterior, estendendo-se da região torácica alta ao sacro. Vinte e quatro pacientes foram submetidos à fixação com sistema de Luque-Galveston (Grupo 1) e 50, com parafusos pediculares (Grupo 2). Foram avaliadas as radiografias pré-operatórias, em tração e no pós-operatório imediato e mediu-se o valor da curva principal do período pré-operatório (Cobb pré), na tração (Cobb tração), e no pósoperatório (Cobb pós), e calculou-se a flexibilidade da curva e a correção final. Também foi calculado o Índice de Cincinnati, que leva em considera-

\section{ABSTRACT}

Objetive: to compare the correction of the major curve and pelvic obliquity using Luque-Galveston instrumentation and pedicle screw constructs in the treatment of neuromuscular scoliosis. Methods: seventy-four patients treated by fusion posterior to the sacrum were investigated using preoperative, traction and postoperative radiographs. Twenty-four cases were submitted to Luque-Galveston instrumentation (Group 1) and fifty were submitted to pedicle screw fixation (Group 2). Radiographic parameters were: major curve angle in the preoperative (Cobb preop), traction (Cobb traction), and postoperative films (Cobb postop), flexibility, final correction, and the Cincinnati Index, which correlates final correction and

\section{RESUMEN}

Objetivo: evaluar el poder de corrección del tornillo pedicular comparado al sistema de Luque-Galveston en el tratamiento quirúrgico de la escoliosis neuromuscular. Métodos: fueron evaluados 74 pacientes sometidos a la artrodesis exclusivamente por la vía posterior, extendiéndose de la región torácica alta a la sacra. De estos pacientes, 24 fueron sometidos a la fijación con sistema de Luque-Galveston (Grupo 1) y 50 con tornillos pediculares (Grupo 2). Fueron evaluadas las radiografias preoperatorias, en tracción y en el postoperatorio inmediato, además de medido el valor de la curva principal preoperatoria (Cobb pre), en tracción (Cobb tracción) y en el postoperatorio (Cobb post). También fue calculada la flexibilidad de la curva y la corrección final. Fue calculado el indice de Cincinnati que lleva en

\footnotetext{
Trabalho realizado pelo Grupo de Escoliose da Associação de Assistência à Criança Deficiente - AACD - São Paulo (SP), Brasil. 
ção a correção final e a flexibilidade (Cincinnati $=$ Correção/Flexibilidade). Os mesmos parâmetros foram calculados para a obliquidade pélvica $(\mathrm{OP})$ : OP pré, OP tração, OP pós, Flexibilidade OP, Correção OP e Índice de Cincinnati para OP. Resultados: a média da idade dos pacientes do Grupo 1 foi de 12,24 anos e do Grupo 2, de 16,13 anos $(p=0,001)$. No Grupo 1 , a principal doença foi a amiotrofia espinhal (38\%) e no Grupo 2, a paralisia cerebral (62\%). O ângulo de Cobb pré foi de $76,67^{\circ}$ para o Grupo 1 e $85,54^{\circ}$ para o Grupo 2. A flexibilidade foi de $45,32 \%$ para o Grupo 1 e $39,47 \%$ para o Grupo 2. A Correção foi de 63,07\% para o Grupo 1 e 59,80\% para o Grupo 2. O índice de Cincinnati para o Grupo 1 foi de 1,44 e de 1,71 para o Grupo 2. Quanto à OP, tivemos OP pré de $20,71^{\circ}$ para o Grupo 1 e $26,60^{\circ}$ para o Grupo 2. A Flexibilidade OP foi de $73,61 \%$ para o Grupo 1 e $56,54 \%$ para o Grupo $2(\mathrm{p}=0,047)$. A Correção OP foi de $73,47 \%$ para o Grupo 1 e de $72,11 \%$ para o Grupo 2. O Índice de Cincinnati da OP foi de 1,09 e 1,49 , respectivamente para os Grupos 1 e $2(p=0,045)$. Conclusões: a instrumentação com parafusos pediculares mostrou correção da escoliose semelhante à fixação com Luque-Galveston e maior poder de correção da obliquidade pélvica no tratamento das deformidades neuromuscluares. flexibility $($ Cincinnati $=$ Correctioln / Flexibility). The same parameters were analyzed for pelvic obliquity (PO): $P O$ preop, $P O$ traction, $P O$ postop, Flexibility PO, Correction $P O$, and Cincinnati Index for PO. Results: mean age in the Group 1 was 12.24 years and 16.13 years in the Group $2(p=0.001)$. The commonest disease in Group 1 was spinal muscular atrophy (38\%) and in Group 2, cerebral palsy (62\%). The mean major curve angle was $76.67^{\circ}$ in Group 1 and $85.54^{\circ}$ in Group 2. Flexibility was $45.32 \%$ in Group 1 and $39.47 \%$ in Group 2. Postoperative correction was $63.07 \%$ in Group 1 and $59.8 \%$ in Group 2. Cincinnati Index was 1.44 in the Group 1 and 1.77 in the Group 2. Mean PO preop was $20.71^{\circ}$ in Group 1 and $26.60^{\circ}$ in Group 2. PO Flexibility was $73.61 \%$ in Group 1 and $56.54 \%$ in Group $2(p=0.047)$. PO Correction was $73.47 \%$ in Group 1 and $72.11 \%$ in Group 2. Cincinnati Index for PO was 1.09 in Group 1 and 1.49 in Group 2 ( $p=0.045)$. Conclusions: postoperative major curve correction was similar with pedicle screws and Luque-Galveston instrumentation, but pelvic obliquity correction was greater when pedicle screw was used in the surgical treatment of neuromuscular scoliosis. consideración la corrección final y la flexibilidad (Cincinnati=corrección/ flexibilidad). Los mismos parámetros fueron calculados para la oblicuidad pélvica (OP): OP pre, OP tracción, $O P$ post, flexibilidad $O P$, corrección $O P$ e indice de Cincinnati para OP. Resultados: el promedio de edad de los pacientes del Grupo 1 fue de 12.24 años y del Grupo 2 de 16.13 años $(p=0.001)$. En el Grupo 1, la principal patología fue la amiotrofia espinal (38\%) y en el Grupo 2, la parálisis cerebral (62\%). O Cobb pre fue de $76.67^{\circ}$ para el Grupo 1 y 85.54 para el Grupo 2. La flexibilidad fue de $45.32 \%$ para el Grupo 1 y $39.47 \%$ para el Grupo 2. La corrección fue de $63.07 \%$ para el Grupo 1 y 59.80\% para el Grupo 2. El indice de Cincinnati para el Grupo 1 fue de 1.44 y de 1.71 para el Grupo 2. Según el OP, tuvimos OP pre de $20.71^{\circ}$ para el Grupo 1 y $26.60^{\circ}$ para el Grupo 2. La flexibilidad OP fue de $73.61 \%$ para el Grupo 1 y 56.54\% para el Grupo 2 ( $p=0.047)$. La corrección OP fue de $73.47 \%$ para el Grupo $1 y$ $72.11 \%$ para el Grupo 2. El índice de Cincinnati de la OP fue de 1.09 y 1.49 , respectivamente para los Grupos 1 y $2(p=0.045)$. Conclusiones: la instrumentación con tornillos pediculares mostró corrección de la escoliosis semejante a la fijación con Luque-Galveston y mayor poder de corrección de la oblicuidad pélvica en el tratamiento de las deformidades neuromusculares.

DESCRIPTORES: Columna vertebral/patología; Escoliosis/cirugía; Fusión vertebral /métodos; Tornillos óseos; Enfermedades neuromusculares/ cirugía; Resultado del tratamiento

\section{INTRODUÇÃO}

As doenças neuromusculares constituem um grupo vasto de patologias que compartilham diversas características, entre elas a alta incidência de deformidades vertebrais ${ }^{1}$.
Normalmente, são curvas de início precoce, de alto valor angular, mais rígidas, colapsantes, de padrão lombar ou toraco-lombar e com obliquidade pélvica² ${ }^{2}$.

Os princípios do tratamento cirúrgico dessas deformidades diferem daqueles da escoliose idiopática. Normalmente, são 
realizadas artrodeses longas que se estendem até a pelve, principalmente nos pacientes não deambuladores devido à perda do equilíbrio ao sentar e à presença de obliquidade pélvica $(\mathrm{OP})^{2}$.

Apesar dos avanços dos materiais de fixação vertebral, o sistema de fios sublaminares por meio da técnica de Luque-Galveston permanece a técnica mais utilizada nesses pacientes. Esta técnica tem se mostrado eficaz ao longo dos anos, com boa taxa de correção e índice de satisfação dos pais e cuidadores ${ }^{3,4}$.

No entanto, nos últimos anos tem-se observado uma tendência à maior utilização dos parafusos pediculares no tratamento das deformidades vertebrais ${ }^{5,6}$, inclusive neuromusculares 7,8 , atribuindo-se a esses instrumentos maior poder de correção, maior estabilidade, menor índice de pseudartrose e menor sangramento ${ }^{9-12}$

O objetivo deste trabalho é comparar o potencial de correção do sistema Luque-Galveston versus parafuso pedicular no tratamento da escoliose neuromuscular em pacientes submetidos à artrodese até o ilíaco exclusivamente pela abordagem posterior.

\section{MÉTODOS}

Foram avaliados, retrospectivamente, os prontuários e radiografias de 74 pacientes com doenças neuromusculares submetidos ao tratamento cirúrgico para escoliose na Associação de Assistência à Criança Deficiente (AACD), São Paulo (SP).

Todos os pacientes foram submetidos à abordagem pela via posterior com artrodese longa da região torácica alta até o sacro. Foram excluídos os casos em que se realizou a via anterior, os casos submetidos à tração ou os que utilizaram qualquer outro tipo de implante que não fosse fio ou parafuso. Também foram excluídos os casos dos pacientes que foram submetidos à cirurgia para correção de deformidades principalmente no plano sagital (hipercifose ou hiperlordose).

Os 74 pacientes foram divididos em dois grupos de acordo com o tipo de implante utilizado: Grupo 1, formado por 24 pacientes nos quais foram utilizados fixação toracolombar com fios sublaminares pela técnica de Luque e fixação pélvica pela técnica de Galveston (Figura 1) e Grupo 2 , formado por 50 pacientes nos quais foram utilizados parafusos pediculares na região torácica e lombar e parafusos de ilíaco para a fixação pélvica. No Grupo 2, em 19 (38\%) dos pacientes foram utilizados ganchos de processo transverso no nível mais cranial da fixação com o objetivo de aumentar a força de arrancamento dos implantes (Figura 2).

A avaliação radiográfica pré-operatória foi realizada por meio de radiografias panorâmicas da coluna vertebral, realizadas com o paciente sentado e com o mínimo apoio possível, obtidas nas incidências em AP e perfil, e por meio de radiografias sob correção com o paciente na posição supina com tração exercida nos membros superiores e inferiores. A avaliação pós-operatória foi realizada através de radiografias panorâmicas feitas no pós-operatório imediato também na incidência em AP e perfil.
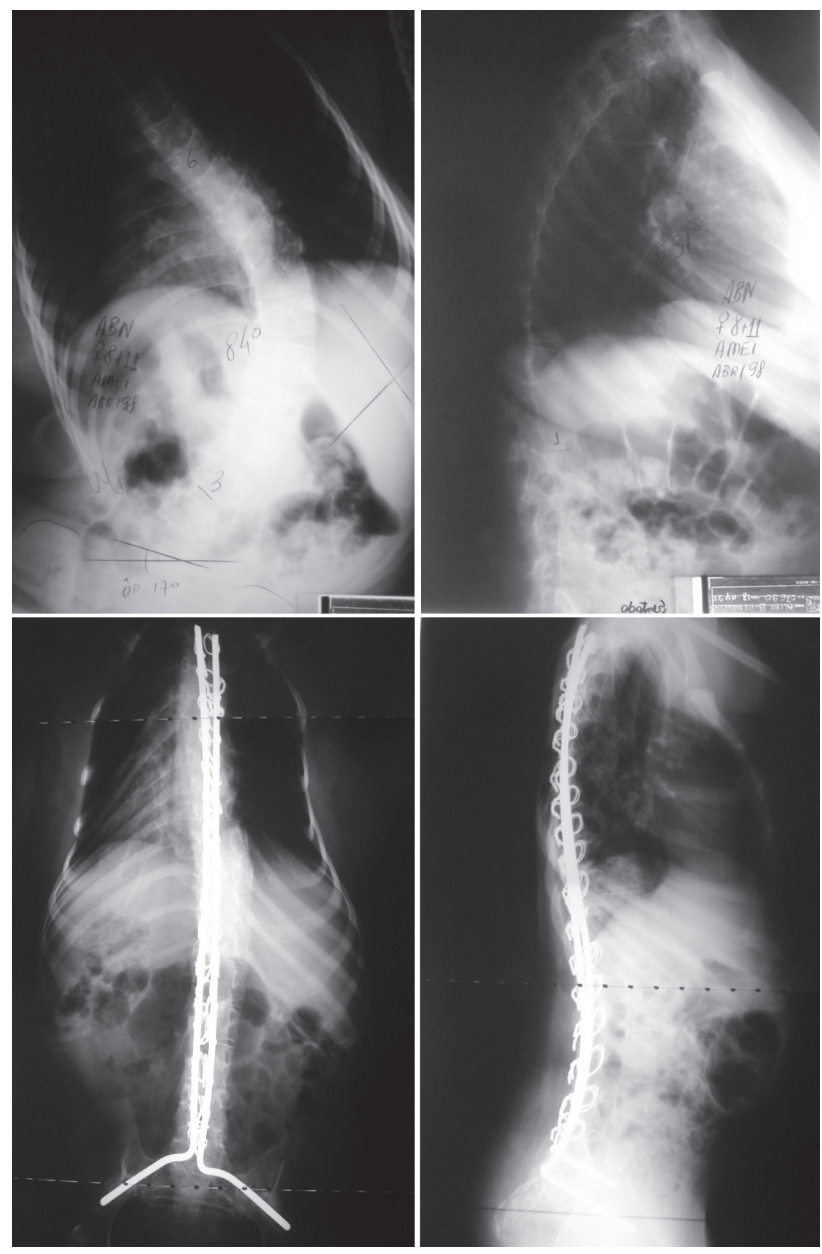

Figura 1

Paciente do Grupo 1, portador de amiotrofia espinhal, submetido à artrodese com instrumental de Luque-Galveston.

Em todos os pacientes, foram mensurados os seguintes parâmetros no plano coronal: padrão da curva, valor angular da curva principal medido pelo método de Cobb na radiografia pré-operatória (Cobb pré), Cobb da curva principal na radiografia sob correção (Cobb tração), Cobb da curva principal na radiografia pós-operatória (Cobb pós), obliquidade pélvica pré-operatória (OP pré), correção da obliquidade pélvica à tração (OP tração) e obliquidade pélvica pós-operatória (OP pós). No plano sagital, foi avaliado apenas o tipo de deformidade associada, já que não era este o objetivo do estudo.

Também foram calculados a flexibilidade da curva principal sob tração (Flexibilidade $=$ Cobb pré - Cobb tração / Cobb pré x 100) e o percentual de correção no pósoperatório (Correção $=$ Cobb pré - Cobb pós / Cobb pré x 100). Para se avaliar o grau de correção obtido no pósoperatório em relação à flexibilidade da curva, foi calculado o Índice de Correção de Cincinnati, conforme descrito por Vora et al. $(\text { Cincinnati }=\text { Correção/Flexibilidade })^{9}$. Este índice permite avaliar o poder de correção com mais precisão, já que a maioria dos estudos ${ }^{5,6,11}$ comparam os 

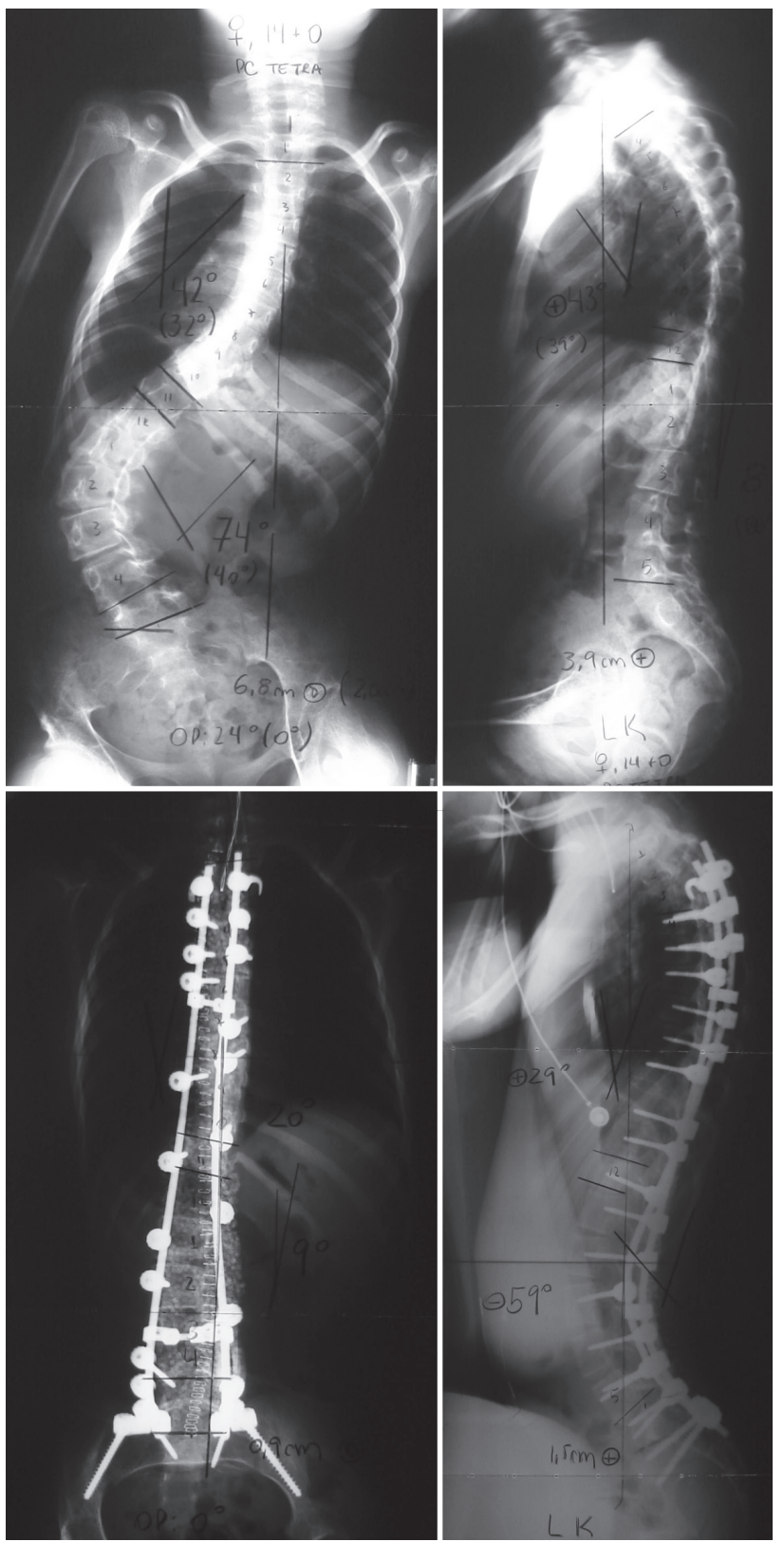

Figura 2

Paciente do Grupo 2, portadora de paralisia cerebral, submetida à artrodese com parafusos pediculares. Note os dois ganchos proximais que não interferem na análise da curva principal.

diversos tipos de implante apenas em relação à correção pós-operatória, sem considerar que a flexibilidade pode ser um fator de confusão nesta análise.

O mesmo foi calculado para a obliquidade pélvica, ou seja, flexibilidade à tração (Flexibilidade $\mathrm{OP}=\mathrm{OP}$ pré - OP tração / OP pré x 100), correção pós-operatória (Correção OP $=$ OP pré - OP pós / OP pré x 100) e adaptou-se o Índice de Cincinnati (Cincinnati $\mathrm{OP}=$ Correção OP / Flexibilidade OP).

A análise estatística foi realizada para comparar os dois grupos por meio do teste de Kruskal-Wallis, utilizando-se o programa Statistical Package for Social Sciences
(SPSS), versão 13.0. O nível de significância considerado foi de $5 \%$.

\section{RESULTADOS}

Dos 74 pacientes avaliados, 41 (55\%) eram do sexo feminino e $33(45 \%)$ do sexo masculino. A média de idade entre os pacientes operados com a técnica de Luque-Galveston (Grupo 1) foi de 12,24 $(\mathrm{dp}=3,37)$ anos, e entre os operados com parafusos pediculares (Grupo 2) de 16,13 (dp=4,53) anos. Quando comparados, o Grupo 1 apresentou média de idade significativamente menor que o Grupo $2(\mathrm{p}=0,001)$.

No Grupo 1, a doença mais frequente foi a amiotrofia espinhal (AME) ( 9 casos, 38\%), seguida da paralisia cerebral (8 casos, 33\%) e lesão medular ( 3 casos, $12 \%)$. Já no Grupo 2, a doença mais frequente foi a paralisia cerebral com 31 casos (62\%), seguida pela amiotrofia espinhal com 4 pacientes (8\%), lesão medular com $4(8 \%)$ e mielomeningocele com 4 (8\%). (Gráfico 1)

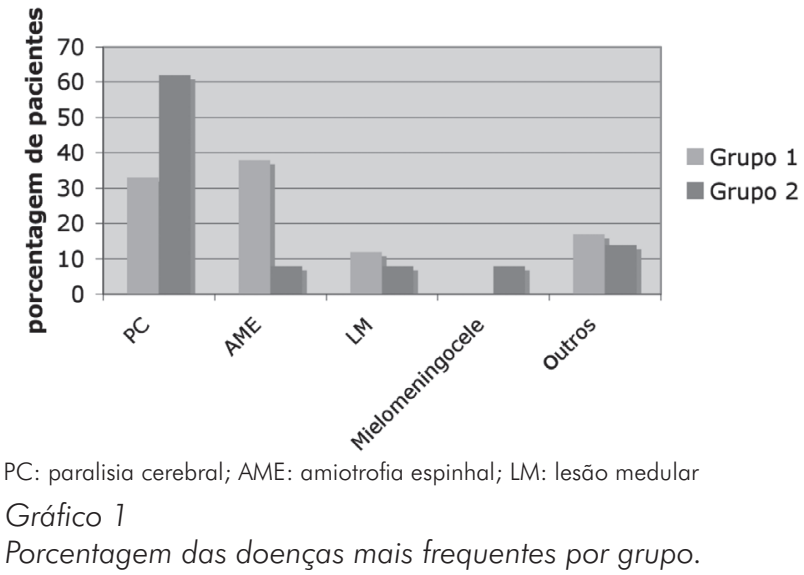

Os tipos de curvas mais frequentes em ambos os grupos foram as toracolombares $(58 \%$ dos pacientes no Grupo $1 \mathrm{e}$ $56 \%$ no Grupo 2), seguidas pelas lombares ( $33 \%$ no Grupo 1 e $36 \%$ no Grupo 2), torácicas (4\% no Grupo 1 e $8 \%$ no Grupo 2 ) e duplas curvas ( $4 \%$ no Grupo 1). Considerou-se apenas a curva principal para o cálculo dos parâmetros analisados.

A média do valor angular da curva principal (Cobb pré) dos pacientes do Grupo 1 foi de 76,67 e no Grupo 2 de $85,54^{\circ}$. A flexibilidade media foi de $45,32 \%$ no Grupo $1 \mathrm{e}$ de $39,47 \%$ no Grupo 2 . A porcentagem de correção foi, em média, $63,07 \%$ e $59,80 \%$, respectivamente para os Grupos 1 e 2. O Índice de Correção de Cincinnati foi, em média, 1,44 e 1,71, também respectivamente para os Grupos 1 e 2. Em três pacientes do Grupo 1 e em sete pacientes do Grupo 2 não foram encontradas as radiografias em tração e, por isso, não foram incluídos no cálculo da flexibilidade e do Índice de Cincinnati (Tabela 1).

$\mathrm{Na}$ análise dos dados referentes à obliquidade pélvica (OP), foram excluídos dez pacientes do Grupo 2 por não possuírem OP na radiografia inicial, porém foram incluídos na avaliação da curva principal por terem sido submetidos à instrumentação até a pelve. 
TABELA 1 - Avaliação radiográfica pré e pós-operatória em relação à curva principal

\begin{tabular}{lccccc}
\hline Variável & Grupo & $\mathbf{n}$ & Média & Desvio padrão & Significância $(\mathbf{p})$ \\
\hline Cobb pré & 1 & 24 & $76,67^{\circ}$ & 20,62 & 0,155 \\
Flexibilidade & 2 & 50 & $85,54^{\circ}$ & 26,02 & 0,116 \\
Correção & 1 & 21 & $45,32 \%$ & 13,51 & \\
& 2 & 43 & $39,47 \%$ & 14,87 & 0,377 \\
Índice de Cincinnati & 1 & 24 & $63,07 \%$ & 13,21 & \\
& 2 & 50 & $59,80 \%$ & 15,63 & 0,141 \\
\hline
\end{tabular}

TABELA 2 - Avaliação radiografia pré e pós-operatória referente à obliquidade pélvica

\begin{tabular}{lccccc}
\hline Variável & Grupo & $\mathrm{n}$ & Média & Desvio padrão & Significância $(\mathbf{p})$ \\
\hline OP pré & 1 & 24 & $20,71^{\circ}$ & 9,39 & 0,112 \\
Flexibilidade OP & 2 & 40 & $26,60^{\circ}$ & 16,34 & \\
& 1 & 21 & $73,61 \%$ & 26,80 & 0,047 \\
Correção OP & 2 & 33 & $56,54 \%$ & 30,37 & 0,871 \\
& 1 & 24 & $73,47 \%$ & 26,55 & \\
Índice de & 2 & 40 & $72,11 \%$ & 25,93 & 0,045 \\
Cincinnati OP & 1 & 21 & 1,09 & 0,69 & 0,81 \\
\hline
\end{tabular}

OP: obliquidade pélvica.

A média da OP pré-operatória foi de $20,71^{\circ}$ no Grupo 1 e de $26,60^{\circ}$ no Grupo 2. A flexibilidade da OP foi de $73,61 \%$ no Grupo 1 e de $56,54 \%$ no Grupo 2. A correção pós-operatória da OP foi de $73,47 \%$ no Grupo 1 e de $72,11 \%$ no Grupo 2. O Índice de Cincinnati foi de 1,09 no Grupo 1 e de 1,49 no Grupo 2. A flexibilidade da OP foi significativamente maior no Grupo $1(\mathrm{p}=0,047)$ e o Índice de Cincinnati da OP significativamente maior no Grupo 2 $(\mathrm{p}=0,045)$. Também foram excluídos da análise da flexibilidade da OP e do Índice de Cincinnati da OP os três casos do Grupo 1 e os sete casos do Grupo 2 cujas radiografias em tração não foram encontradas (Tabela 2).

Quanto ao plano sagital, a principal deformidade associada era a cifose por colapso ou cifose aparente devido à gravidade da rotação, como descrito por Stagnara ${ }^{13}$.

\section{DISCUSSÃO}

O tratamento cirúrgico das escolioses neuromusculares é classicamente realizado por meio de fusões longas, estendendo-se até a pelve com fixação através da técnica de Luque-Galveston ${ }^{1-4}$.

No entanto, com o advento dos parafusos pediculares, vários estudos têm sido realizados para demonstrar a sua segurança, mesmo quando utilizados na região torácica para correção de deformidades ${ }^{10,14}$. Com isso, existe a tendência em utilizar cada vez mais esse tipo de implante, inclusive nas deformidades de alto valor angular, como é frequente nas escolioses neuromusculares ${ }^{7,8,15}$.

Algumas das vantagens que se atribui ao parafuso pedicular é que ele permite maior poder de correção, menor perda desta ao longo do tempo, diminui a necessidade de liberações por via anterior, possui melhor correção no plano sagital, menor sangramento, fica completamente intraósseo, é uma fixação mais rígida diminuindo o risco de pseudartrose e diminui a necessidade de imobilização pós-operatórias ${ }^{5,10-12,15,16}$. A sua principal desvantagem seria o custo elevado, mas existe um estudo que mostra que o custo do implante representa apenas $5 \%$ do custo total do tratamento do paciente com escoliose idiopática ${ }^{17}$, e esta porcentagem deve ser bem inferior quando se consideram pacientes com doenças neuromusculares.

Este trabalho demonstrou maior correção da curva principal nos pacientes operados com a técnica de LuqueGalveston em relação ao grupo operado com parafusos (63,07 versus 59,80\%). Apesar do resultado não esperado, uma análise mais detalhado mostra que os pacientes operados com parafusos possuíam curvas de maior magnitude $\left(85,54\right.$ versus $\left.76,67^{\circ}\right)$ e menor flexibilidade à tração $(39,47$ versus 45,32\%). Comparando-se os Índices de Cincinanti, obtiveram-se valores mais elevados para o grupo dos pacientes operados com parafusos $(1,71 \mathrm{ver}$ sus 1,44$)$. Nenhum desses resultados foi estatisticamente significativo.

A idade e a doença de base são outros fatores que podem explicar esses resultados. Os pacientes operados com parafusos apresentavam idade significativamente maior, ou seja, possivelmente com maior retração dos tecidos e maior estruturação da deformidade. A doença mais frequente neste grupo foi a paralisia cerebral, que apresenta um componente de espasticidade importante. Os pacientes operados com fios eram, na maioria, portadores de amiotrofia espinhal, que se caracteriza por hipotonia. 
Watanabe e Lenke compararam o tipo de implante utilizado na região apical da curva em 68 pacientes com deformidades de alto valor angular, maior que $100^{\circ}$. Destes, 44 eram escolioses neuromusculares. Neste estudo, eles observaram que o parafuso pedicular demonstrou maior poder de correção no plano coronal (61,0 versus $51,8 \%)$ e menor perda desta ao longo do tempo em relação ao fio sublaminar. Também demonstrou menor incidência de abordagem por via anterior dentre os pacientes do grupo fixado com parafusos. Quando se compararam isoladamente os pacientes submetidos à cirurgia apenas pela via posterior, nos 11 pacientes do grupo com fio houve correção de $46 \%$ e nos dez pacientes do grupo com parafuso houve correção de $71 \%$. Uma das explicações para esta taxa de correção elevada é que em cinco casos de artrodese apenas pela via posterior com parafusos foram realizadas osteotomias $^{18}$.

No estudo realizado por Bulman, em quinze pacientes portadores de paralisia cerebral submetidos à fixação com sistema tradicional de Luque-Galveston, obtevese uma correção média de $48,6 \%{ }^{19}$. Comstock, também avaliando pacientes com paralisia cerebral tratados com Luque-Galveston, mostrou uma correção de 51\% quando se realizou o procedimento apenas pela via posterior, e de $57 \%$ quando se associou à liberação anterior ${ }^{20}$. Em ambos os casos, os resultados da presente pesquisa foram superiores.

Ao se utilizarem parafusos pediculares para deformidades neuromusculares, observa-se uma média de correção de $60 \% \%^{7,8}$, condizente com os nossos resultados.
Quando se analisou a OP, também foram obtidos valores levemente superiores no grupo com fixação tipo LuqueGalveston (73,47 versus $72,11 \%)$. No entanto, quando se observou o Índice de Cincinnati para a OP, houve maior correção com os parafusos de ilíaco estatisticamente significativa $(1,09$ versus 1,49 , com $\mathrm{p}=0,045)$. Isso ocorreu porque, no Grupo 1 , as deformidades eram de menor valor angular e mais flexíveis $(73,61$ versus $56,54 \%, \mathrm{p}=0,047)$.

Peelle também comparou a fixação pélvica por meio da haste de Galveston com parafusos ilíacos. Neste estudo, os parafusos também apresentaram maior poder de correção no pós-operatório imediato (73 versus $66 \%$ ) e menor lise peri-implante ao longo do seguimento ${ }^{21}$.

O principal objetivo do tratamento cirúrgico da escoliose neuromuscular é obter um tronco compensado sobre uma pelve nivelada. Com isso, instrumentais que possuam maior poder de correção da OP podem ser úteis para melhorar o tratamento desses pacientes. Além disso, apesar de não ser o fator mais importante a ser analisado, materiais que possuem maior poder de correção da escoliose podem ser importantes, principalmente ao diminuir a necessidade de abordagem pela via anterior, o que é ainda mais importante nos pacientes neuromusculares que, normalmente, já possuem uma capacidade pulmonar diminuída.

\section{CONCLUSÕES}

No tratamento cirúrgico da escoliose neuromuscular, o parafuso pedicular apresentou poder de correção da curva principal semelhante ao sistema de Luque-Galveston, porém com maior poder de correção da obliquidade pélvica.

\section{REFERÊNCIAS}

1. Berven S, Bradford DS.

Neuromuscular scoliosis: causes of deformity and principles for evaluation and management. Semin Neurol. 2002;22(2):167-78.

2. Lonstein JE. Neuromuscular spinal deformity. In: Weinstein SL, editor. The pediatric spine: principles and practice. 2nd ed. Philadelphia: Lippincott Williams \& Wilkins; 2001. p. 789-96.

3. Lonstein JE, Akbarnia A. Operative treatment of spinal deformities in patients with cerebral palsy or mental retardation. An analysis of one hundred and seven cases. J Bone Joint Surg Am. 1983;65(1):43-55.

4. Lonstein JE. Spine deformity due to cerebral palsy. In: Weinstein SL, editor. The pediatric spine: principles and practice. 2nd ed. Philadelphia: Lippincott Williams \& Wilkins; 2001. p. 797-808.
5. Kim YJ, Lenke LG, Cho SK, Bridwell $\mathrm{KH}$, Sides B, Blanke K. Comparative analysis of pedicle screw versus hook instrumentation in posterior spinal fusion of adolescent idiopathic scoliosis. Spine. 2004;29(18):2040-8.

6. Cheng I, Kim Y, Gupta MC, Bridwell $\mathrm{KH}$, Hurford RK, Lee SS, et al. Apical sublaminar wires versus pedicle screw - which provides better results for surgical correction of adolescent idiopathic scoliosis? Spine. 2005;30(18):2104-12.

7. Modi HN, Suh SW, Song HR, Fernandez HM, Yang JH. J Orthop Surg Res 2008;3:23-31.

8. Oliveira GC, Cavali PTM, Landim E, Santos MAM, Lehoczki MA. Instrumental de $3^{\text {a }}$ geração no tratamento de escoliose em pacientes com paralisia cerebral tetraparéticos - análise dos resultados clínico e radiográfico. Coluna/Columna. 2007;6(4):201-10.
9. Vora V, Crawford A, Babekhir N, Boachie-Adjei O, Lenke L, Peskin M, et al. A pedicle screw construct gives an enhanced posterior correction of adolescent idiopathic scoliosis when compared with other constructs: myth or reality. Spine. 2007;32(17):1869-74. Comment in: Spine. 2008;33(1):120; author reply 120-1.

10.Suk SI, Lee Ck, Kim WJ, Chung YJ, Park YB. Segmental pedicle screw fixation in the treatment of thoracic idiophatic scoliosis. Spine. 1995;20(12):1399-405.

11. Kim YJ, Lenke LG, Kim J, Bridwell $\mathrm{KH}$, Cho SK, Cheh G, et al. Comparative analysis of pedicle screw versus hybrid instrumentation in posterior spinal fusion of adolescent idiopathic scoliosis. Spine. 2006;31(3):291-8. 
12.Liljenqvist U, Lepsien U, Hackenberg L, Niemeyer T, Halm H. Comparative analysis of pedicle screw and hook instrumentation in posterior correction and fusion of idiopathic thoracic scoliosis. Eur Spine J. 2002;11(4):336-43.

13.Stagnara P. Traitement chirurgical des scolioses cyphosantes chez l'adulte. Acta Orthop Belg. 1981;47(4-5): 721-39.

14.Kim YJ, Lenke LG, Bridwell KH, Cho YS, Riew D. Free hand pedicle screw placement in the thoracic spine: is it safe? Spine. 2004;29(3):333-42; discussion 342.

15.Luhmann SJ, Lenke LG, Kim YJ, Bridwell KH, Schootman M. Thoracic adolescent idiopathic scoliosis curves between 70 degrees and 100 degrees: is anterior release necessary? Spine. 2005;30(18):2061-7.
16.Lenke LG, Kuklo TR, Ondra S, Polly DW Jr. Rationale behind the current state-of-the-art in the treatment of scoliosis (in the pedicle screw era). Spine. 2008;33(10):1051-4. Comment on: Spine. 2007;32(24):2641-3.

17.Storer SK, Vitale MG, Hyman JE, Lee FY, Choe JC, Roye DP Jr. Correction of adolescent idiopathic scoliosis using thoracic pedicle screw fixation versus hook constructs. J Pediatr Orthop. 2005;25(4):415-9.

18. Watanabe K, Lenke LG, Bridwell KH, Kim YJ, Watanabe K, Kim YW, et al. Comparison of radiographic outcomes for the treatment of scoliotic curves greater than 100 degrees: wires versus hooks versus screws. Spine. 2008;33(10):1084-92.

19.Bulman WA, Dormans JP, Ecker ML, Drummond DS. Posterior spinal fusion for scoliosis in patients with cerebral palsy: a comparison of Luque rod and Unit Rod instrumentation. J Pediatr Orthop. 1996;16(3):314-23.
20.Comstock CP, Leach J, Wenger DR. Scoliosis in total-body-involvement cerebral palsy. Analysis of surgical treatment and patient and caregiver satisfaction. Spine. 1998;23(12):141224; discussion 1424-5.

21.Peelle MW, Lenke LG, Bridwell KH, Sides B. Comparison of pelvic fixation techniques in neuromuscular spinal deformity correction: Galveston rod versus iliac and lumbosacral srews. Spine. 2006;31(20):2392-8; discussion 2399.

\section{Correspondência}

Murilo Tavares Daher

Rua Martinico Prado, 71, apto. 24 Vila Buarque

CEP: 01224-010 - São Paulo (SP), Brasil

Tel.: (1 1) 7542-6270/(62) 3281-2397

E-mail: murilodaher@uol.com.br 